\title{
Exendin-4 ameliorates high glucose- induced fibrosis by inhibiting the secretion of miR-192 from injured renal tubular epithelial cells
}

\author{
Yijie Jia', Zongji Zheng ${ }^{1}$, Meiping Guan ${ }^{1}$, Qian Zhang ${ }^{1}$, Yang Li' ${ }^{1}$ Ling Wang ${ }^{1}$ and Yaoming Xue ${ }^{1}$
}

\begin{abstract}
Extracellular vesicles (EVs), which contain microRNA (miRNA), constitute a novel means of cell communication that may contribute to the inevitable expansion of renal fibrosis during diabetic kidney disease (DKD). Exendin- 4 is effective for treating DKD through its action on GLP1R. However, the effect of exendin-4 on EV miRNA expression and renal cell communication during the development of DKD remains unknown. In this study, we found that EVs derived from HK-2 cells pre-treated with exendin-4 and high glucose $(\mathrm{Ex}-\mathrm{HG})$, which were taken up by normal HK-2 cells, resulted in decreased levels of FN and Col-I compared with EVs from HK-2 cells pre-treated with HG alone. Furthermore, we found that pretreatment with HG and exendin-4 may have contributed to a decrease in miR-192 in both HK-2 cells and EVs in a p53-dependent manner. Finally, we demonstrated that the amelioration of renal fibrosis by exendin- 4 occurred through a miR-192-GLP1R pathway, indicating a new pathway by which exendin-4 regulates GLP1R. The results of this study suggest that exendin-4 inhibits the transfer of EV miR-192 from HG-induced renal tubular epithelial cells to normal cells, thus inhibiting GLP1R downregulation and protecting renal cells. This study reports a new mechanism by which exendin-4 exerts a protective effect against DKD.
\end{abstract}

\section{Introduction}

With the increase in the prevalence of diabetes mellitus, diabetic kidney disease (DKD) has become the leading cause of chronic kidney disease worldwide ${ }^{1}$. One of the most common characteristics of DKD is tubulointerstitial fibrosis, which accelerates renal failure and appears early in diabetic kidney injury ${ }^{2,3}$. A previous study indicated that hyperglycemia can induce extracellular matrix accumulation of renal tubular epithelial cells, which is a vital step in tubulointerstitial fibrosis ${ }^{4-6}$.

Studies have reported that injured renal tubular epithelial cells can influence normal cells and other resident renal cells through the release of extracellular vesicles

Correspondence: Yaoming Xue (xueyaoming999@126.com)

'Department of Endocrinology \& Metabolism, Nanfang Hospital, Southern Medical University, Guangzhou, China

These authors contributed equally: Yijie Jia, Zongji Zheng.
$(\mathrm{EVs})$, resulting in a vicious cycle of renal fibrosis ${ }^{7,8}$. EVs, which contain proteins, mRNA, and microRNA (miRNA), reflect a newly discovered method of cell-to-cell communication $^{9,10}$. Existing research indicates that EVs can distribute miRNA among cells, thereby promoting disease progression $^{11,}{ }^{12}$. However, the role of EV-mediated miRNA delivery in the progression of DKD remains unclear.

Exendin-4, a long-acting GLP-1 analog, has been used for the treatment of type 2 diabetes mellitus. GLP-1 exerts its biological action by binding to its specific receptor, the GLP-1 receptor (GLP1R), which is present in various organs, such as the liver, brain, and kidney ${ }^{13,14}$. In addition to directly targeting GLP1R, exendin- 4 has been indicated by many studies to potentially function through other mechanisms. Lee et al. ${ }^{15}$ reported that the levels of several miRNAs in the pancreas were altered after 
treatment with exendin-4, suggesting that exendin-4 may exert its function through miRNA; however, the mechanism remains unclear. p53, a transcription factor that promotes DKD progression ${ }^{16}$ and regulates several miRNAs, is reportedly downregulated by exendin $-4^{17}$. Thus, we propose that exendin- 4 may regulate miRNA expression through p53.

In this study, we aimed to examine the effects of exendin- 4 on miRNA expression in renal tubular epithelial cells and in the EVs from these cells. We also determined whether exendin-4 influences EV miRNA delivery from high glucose (HG)-treated renal tubular epithelial cells to normal ones and determined the underlying mechanisms.

\section{Materials and methods}

\section{Cell culture and treatment}

The human renal tubular epithelial cell line HK-2 (ATCC, Manassas, USA) was cultured in Dulbecco's modified Eagle's medium with $5.6 \mathrm{mM}$ glucose (NG) supplemented with $10 \%$ fetal bovine serum (FBS; Gibco, Australia). The cells were incubated in a $5 \% \mathrm{CO}_{2}$ incubator at $37^{\circ} \mathrm{C}$. When HK-2 cells were seeded at $\sim 60 \%$ confluence, they were cultured in 2\% FBS DMEM for $24 \mathrm{~h}$ and subsequently exposed to DMEM-containing $30 \mathrm{mM}$ glucose (HG) and exendin- $4(0,0.1,1,10$, or $100 \mathrm{nM})$ for an additional $48 \mathrm{~h}$.

For cell transfection, cells were transfected with miR192 mimic, miR-192 inhibitor or GLP1R siRNA, and the appropriate negative controls (Ribo, China) at a concentration of $50 \mathrm{~nm}$, and seeded at $60 \%$ confluence using Lipofectamine 3000 (Invitrogen, CA, USA) according to the manufacturer's protocol. For co-culture experiments, EVs isolated from donor cells were added to recipient cells at a concentration of $50 \mu \mathrm{g} / \mathrm{ml}$.

Cells were harvested $48 \mathrm{~h}$ after transfection or coculture.

\section{EV extraction}

HK-2 cells were cultured in DMEM medium with 5.5 $\mathrm{mM}$ D-glucose and 10\% FBS until they reached $60 \%$ confluence. Subsequently, the media was changed to DMEM with $5.5 \mathrm{mM}$ D-glucose, $30 \mathrm{mM}$ D-glucose, or 30 $\mathrm{mM}$ D-glucose with $10 \mathrm{nM}$ exendin- 4 or transfected with the miR-192 inhibitor in 2\% EV-depleted FBS for $48 \mathrm{~h}$. EVs were removed from FBS using a previously reported procedure $^{18}$. The culture supernatants were collected and centrifuged at $3000 \times g$ for $15 \mathrm{~min}$ to remove cells and cell debris. Subsequently, one-third volume of ExoQuick-TC (System Biosciences) was added to the supernatants and mixed. After $24 \mathrm{~h}$ of refrigeration, the mixture was centrifuged at $1500 \times g$ for $30 \mathrm{~min}$, and the supernatant was removed. EVs were suspended in $30 \mu \mathrm{l}$ of PBS and were stored at $-80^{\circ} \mathrm{C}$ for further analysis.

\section{Transmission electron microscopy (TEM)}

For TEM, $20 \mu \mathrm{l}$ of a fresh EV sample was placed on a 200-mesh-nickel grid, excess fluid was removed, and a drop of $2 \%$ phosphotungstic acid was transferred to the grid for $5 \mathrm{~min}$ for staining. Samples were analyzed with a JEOL JEM-1400 transmission electron microscope.

\section{Fluorescent labeling of EVs}

EVs $(50 \mu \mathrm{g})$ suspended in $160 \mu \mathrm{l}$ of PBS were incubated in $20 \mu \mathrm{l} \mathrm{of} 5 \mu \mathrm{g} / \mathrm{ml}$ Dil-C18 at room temperature for 30 min. EVs were washed with PBS and harvested by centrifugation $(200,000 \times g$ for $1 \mathrm{~h})$. Subsequently, EVs were resuspended in $5.5 \mathrm{mM}$ D-glucose DMEM and incubated with recipient cells at a concentration of $50 \mu \mathrm{g} / \mathrm{ml}$ for 24 h. Next, the cells were washed twice, stained with DAPI, and observed under an Olympus microscope (Japan).

\section{RNA isolation and reverse transcription quantitative PCR}

Total RNA was prepared from cells using TRIzol reagent (Takara, Dalian, China) according to the manufacturer's instructions. RNA quality was measured using a NanoDrop ND-1000 spectrophotometer (Thermo Fisher Scientific, Wilmington, DE, USA).

Approximately $500 \mathrm{ng}$ of RNA was used for cDNA synthesis with a Takara Prime Script ${ }^{\circledast}$ RT reagent kit (Takara, Dalian, China). SYBR Premix Ex Taq (Takara) was used to quantify mRNA expression, and $\beta$-actin was used as an internal control. Primers for GLP1R, fibronectin (FN), type I collagen (Col-I), and $\beta$-actin were synthesized by Invitrogen (Shanghai, China) as follows: GLP1R: forward 5'-TCAAGGTCAACGGCTTATTAGTGAA-3', reverse 5'-CCCAAGTGATGCAAGCAGAG-3'; $\mathrm{FN}$ : forward 5'-TAGCCCTGTCCAGGAGTTCA-3', reverse 5'-CTGCAAGCCTTCAATAGTCA-3'; Col-I: forward 5'-GCAGGAGGTTTCGGCTAAGT-3', reverse $5^{\prime}$-GCAACAAAGTCCGCGTATCC-3'; and $\beta$-actin: forward $5^{\prime}$-CCCTGGACTTCGAGCAAGAGAT-3', reverse 5'-GTTTTCTGCGCAAGTTAGG-3'.

EV miRNA was extracted using a miRcute miRNA isolation kit (TianGen, China). A miRcute miRNA firststrand cDNA synthesis kit (TianGen, China) was used for small RNA reverse transcription. Subsequently, a miRcute miRNA qPCR detection kit (TianGen, China) was used to quantify miRNA expression; U6 was used as an internal control. Primers for miR-192 and U6 were purchased from TIANGEN.

RT-PCR was performed using a LightCycler 480 RealTime PCR System (Roche; Hoffmann-La Roche Ltd, Basel, Switzerland). The fold-change of each mRNA/miRNA was calculated using the $2^{-\triangle \triangle \mathrm{Ct}}$ method.

\section{Western blotting}

Total protein was extracted with RIPA lysis buffer (KeyGEN Biotech, China), and protein concentrations 
were measured using the BCA method (Takara Biotechnology, Japan). Subsequently, $50 \mu \mathrm{g}$ of protein was separated on a $10 \%$ SDS-PAGE gel and transferred to a PVDF membrane (Merck Millipore, MA, USA). Membranes were blocked at room temperature for $1 \mathrm{~h}$ and then incubated at $4{ }^{\circ} \mathrm{C}$ overnight with primary antibodies for FN (Sigma, St. Louis, MO, USA), Col-I (Merck Millipore, Germany), GLP1R (Abclonol, USA), p53 (Cell Signaling, MA, USA), CD63 (Santa Cruz, CA, USA), and $\beta$-actin (Santa Cruz, CA, USA). After an additional incubation with a fluorescent secondary antibody at room temperature for $1 \mathrm{~h}$ (1:15,000; LI-COR Biosciences, Lincoln, NE, USA), images were visualized with an Odyssey infrared imaging system (LI-COR, Lincoln, NE, USA), and densitometry analysis was performed using Quantity One software (Bio-Rad, USA).

\section{Luciferase assay}

To create the GLP1R 3'-UTR luciferase reporter plasmid, a pMIR-REPORT vector (Ambion, USA) was used, and nucleotides 262-461 of the GLP1R 3'-UTR were cloned into the plasmid. A similar plasmid with a mutated miR-192 seed sequence (from AGGTCAA to CATGTGC) was also constructed using a site-directed mutagenesis kit (TianGen, China).

HK-2 cells were seeded in a 24-well plate and transfected with a mixture of $1 \mu \mathrm{g}$ of pMIR-REPORT plasmid, $0.2 \mu \mathrm{g}$ of $\beta$-gal plasmid (Ambion, USA), and $50 \mathrm{nmol}$ miR-192 or negative control (NC) mimic and inhibitor with Lipofectamine 3000 (Invitrogen, CA, USA). The cells were lysed $48 \mathrm{~h}$ after transfection, and luciferase activity was measured with a luciferase assay kit (Beyotime, China). All experiments were repeated three times.

\section{Chromatin Immunoprecipitation (CHIP) analysis}

A ChIP-IT ${ }^{\circ}$ Express kit (Active Motif, Bedford, MA) was used to perform the ChIP assay. HK-2 cells were treated with NG, HG or HG + exendin- 4 for $48 \mathrm{~h}$ and cross-linked using $1 \%$ formaldehyde in DMEM; the crosslink reaction was stopped by glycine. Samples were collected, resuspended, and sheared to $800 \mathrm{bp}$. Subsequently, $2 \mu \mathrm{g}$ anti-p53 and $2 \mu \mathrm{g}$ IgG antibody (Cell Signaling, MA, USA) was used for immunoprecipitation. Input DNA was evaluated and subjected to RT-PCR according to the manufacturer's instructions. Briefly, a standard curve was made by performing qPCR with a primer targeting known DNA quantities of input DNA. Subsequently, ChIP Ct and IgG Ct values were used to calculate the ChIP DNA and IgG quantity. Fold enrichment was calculated as ChIP DNA quantity/IgG DNA quantity. Primers for the p53-binding sites on the miR192 promoter region were designed according to a previous manuscript ${ }^{19}$.

\section{EV exendin-4 dosage by high-performance liquid chromatography (HPLC)}

EVs were pre-treated for HPLC measurement using the methods reported by Pascucci et al. ${ }^{20}$ Briefly, EV samples were added to equal volumes of $0.6 \mathrm{~N}$ perchloric acid, mixed and centrifuged at $200 \times g$ at $4{ }^{\circ} \mathrm{C}$ for $10 \mathrm{~min}$, followed by filtration and injection into HPLC. An Agilent 1260 system combined with diode-array detection (DAD) was used for HPLC analysis of these samples. An Agilent C18 column $(5 \mu \mathrm{m}, 250 \mathrm{~mm} \times 4.6 \mathrm{~mm})$ with an HPLC guard cartridge system (Phenomenex, SecurityGuard) was used for HPLC analysis. The mobile phases consisted of acetonitrile (A) and $20 \mathrm{mmol} / \mathrm{l} \mathrm{KH}_{2} \mathrm{PO}_{4}$ with $\mathrm{pH}$ adjusted to 3 with phosphoric acid, (B) using an isocratic elution program of $37 \%$ A for $0-30 \mathrm{~min}$. The flow rate was 1.0 $\mathrm{ml} / \mathrm{min}$, and column temperature was set to $30^{\circ} \mathrm{C}$. The DAD detector was set at $210 \mathrm{~nm}$.

\section{Statistical analyses}

All data are presented as the mean \pm SEM. Student's $t$ test and one-way ANOVA were used to determine statistical significance. $P$-values $<0.05$ were considered statistically significant.

\section{Results}

\section{Exendin-4 alleviates HG-induced fibrosis in HK-2 cells}

To determine the effects of exendin-4 on HK-2 cell fibrosis under HG conditions, we treated HK-2 cells with various concentrations of exendin- 4 for 24,48 , or $72 \mathrm{~h}$. Treatment with HG significantly increased the levels of FN and Col-I mRNA, whereas large doses of exendin-4 (10 and $100 \mathrm{nM}$ ) decreased FN and Col-I expression (Fig. 1a-f). In addition, treatment with $10 \mathrm{nM}$ exendin- 4 for $48 \mathrm{~h}$ also decreased HG-induced FN and Col-I expression at the protein level (Fig. 1g, h)

\section{Exendin-4 reverses the production of EVs induced by HG treatment}

To explore the role of HG and exendin-4 on EV release, we first isolated EVs from renal tubular epithelial cells. TEM revealed that EVs appeared as a cluster of cupshaped vesicles (Fig. 2a), and immunoblotting confirmed the expression of CD63 (Fig. 2b).

To explore the effect of exendin- 4 on EV release, we used total EV protein/total cell number to evaluate the production of EVs. The HG group had a significantly greater total number of EVs than the control group. The addition of exendin-4 to the HG group (Ex-HG) significantly reversed EV production (Fig. 2c).

\section{EVs derived from exendin-4 and HG-pre-treated (Ex-HG) donor cells do not promote fibrosis in recipient cells}

EVs from injured renal tubular epithelial cells have been reported to promote the fibrosis in recipient cells ${ }^{8}$. 

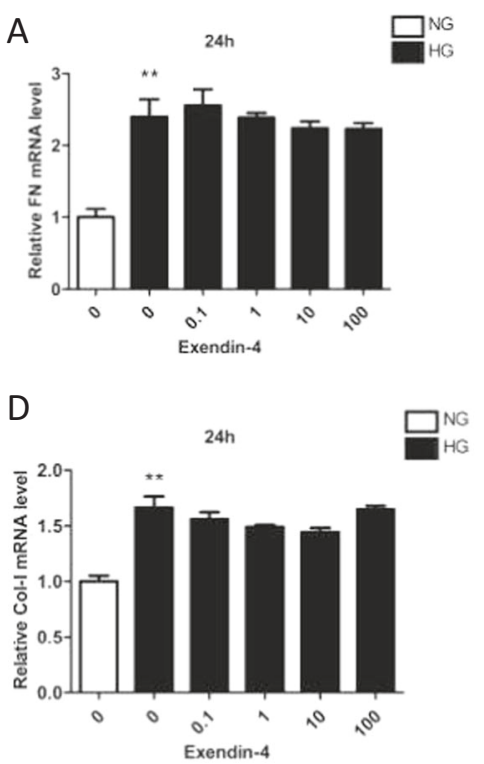

G

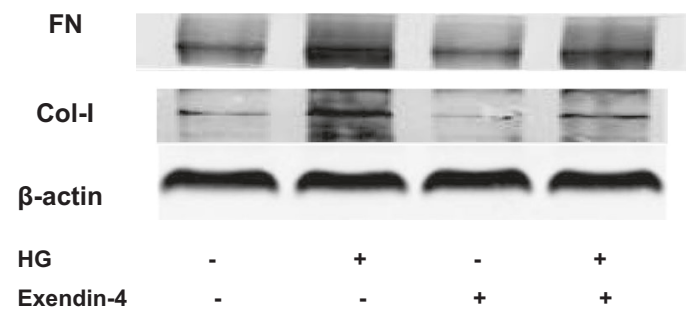

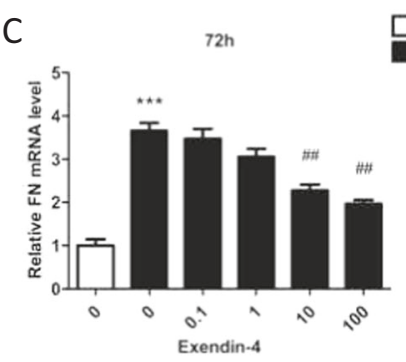

$E$

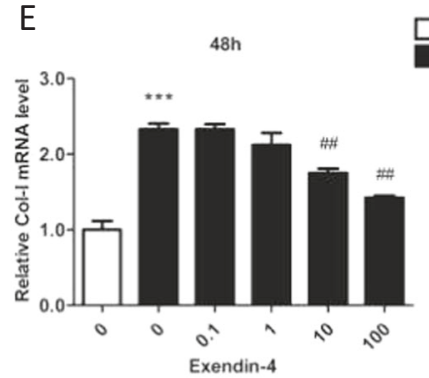

$\mathrm{F}$

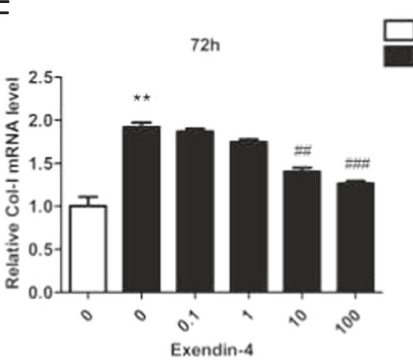

Fig. 1 Effect of exendin-4 on fibrosis in HG-induced HK-2 cells. HK-2 cells were cultured in DMEM with 5.6 mM glucose (NG) or 30 mM glucose $(\mathrm{HG})$ and different concentrations of exendin-4. a-f Relative expression of FN and Col-I in HK-2 cells after incubation with HG and various concentrations of exendin-4 for 24, 48 , and 72 h. $\mathbf{g}, \mathbf{h}$ FN and Col-I expression in HK-2 cells after incubation with HG and 10 nM exendin-4 for 48 h was analyzed by western blot ( ${ }^{* *} P<0.01$ and ${ }^{* * *} P<0.001$ versus the NG group; ${ }^{\#} P<0.05,{ }^{\# \#} P<0.01$, and ${ }^{\# \#} P<0.001$ versus the HG group)

Because exendin-4 alleviates HG-induced fibrosis, we examined whether EVs from Ex-HG-treated donor cells led to fibrosis in recipient cells. To confirm that EVs can be transported into recipient cells, we labeled EVs with Dil-C18. After $24 \mathrm{~h}$ of incubation, Dil-C18 appeared in the cytoplasm of recipient cells (Fig. $2 \mathrm{~d}-\mathrm{f}$ ), which indicated that EVs were transported into the recipient cells.

Furthermore, EVs from the NG, HG, exendin-4, and ExHG groups were collected and co-cultured with HK-2 cells. As expected, the EVs derived from the HG group markedly increased the levels of FN and Col-I, but the addition of exendin-4 to donor cells (Ex-HG) resulted in decreased levels of FN and Col-I in recipient cells compared with HG group-derived EVs (Fig. $2 \mathrm{~g}-\mathrm{j}$ ).

To determine whether the ability of EVs from the ExHG group to alleviate the fibrosis effect on HK-2 cells was mediated by exendin- 4 within the EVs, we measured the concentration of exendin-4 in EVs derived from exendin- 4-treated HK-2 using HPLC. As shown in Fig. 3a-c, the HPLC chromatograms from EVs derived from exendin-4treated HK-2 cells did not exhibit a peak at the identical retention time as the peak from the standard sample of exendin- 4 in PBS $(1 \mathrm{mg} / \mathrm{ml})$, suggesting that little exendin-4 was packaged into EVs from the exendin-4 group. Further, after we co-cultured HG-treated HK-2 cells with EVs from exendin-4-treated HK-2 cells, we did not observe changes in FN or Col-I expression (Fig. 3d, e).

\section{Exendin-4 downregulates HG-induced miR-192 expression in EVs}

miRNA has been reported to be important in EVmediated cell-to-cell communication. Thus, we proposed that the EVs derived from the Ex-HG group alleviated the fibrosis effect on HK-2 cells through miRNA. p53 has a vital role in regulating miRNA expression, and the miR192 family, miR-29a, miR-34a, and miR-199, are 
A

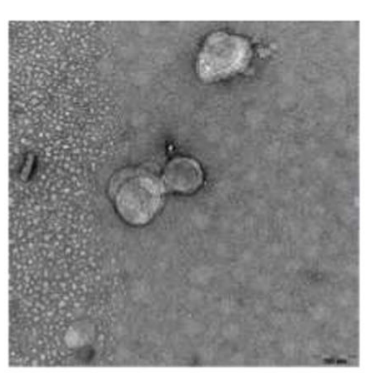

$\mathrm{E}$
B

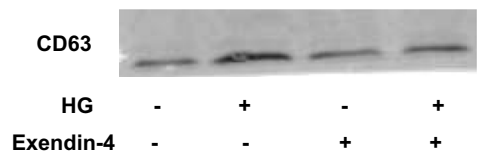

$\mathrm{F}$

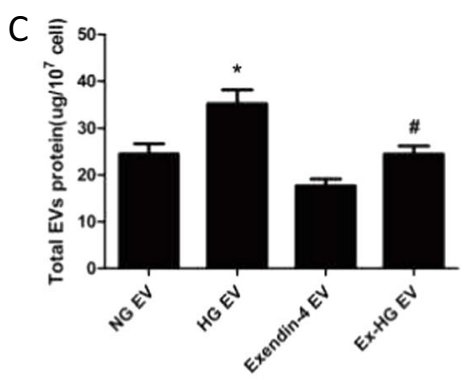

G

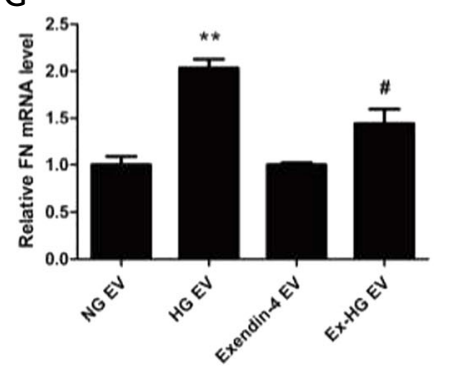

J

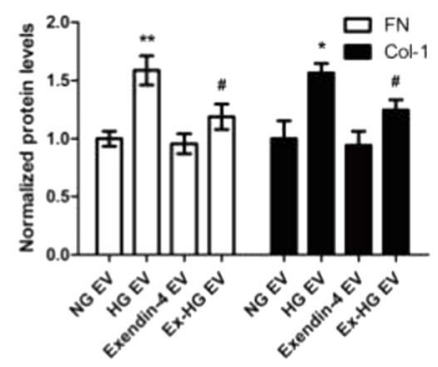

Fig. 2 Treatment of HK-2 cells with Ex-HG group-derived EVs induced less fibrosis in recipient cells than treatment with HG group-derived EVs. a EVs from HK-2 cells identified using TEM. b Western blot of CD63 expression of in EVs from $5 \times 10^{7}$ cells. c Total EV protein from the NG group, HG group, exendin-4 group, and Ex-HG group. $\mathbf{d}-\mathbf{f}$ Co-culture of EVs with recipient cells. EVs from donor cells were labeled with Dil-C18, and recipient cells were labeled with DAPl; red represents Dil-C18, and blue represents nuclear DNA. $\mathbf{g}, \mathbf{h}$ Relative expression of FN and Col-I in HK-2 cells after incubation with EVs derived from NG, HG, exendin-4, and Ex-HG groups for $48 \mathrm{~h}$. $\mathbf{i}, \mathbf{j}$ The FN and Col-I expression in HK-2 cells after incubation with EVs derived from NG, HG, exendin-4, and Ex-HG groups for $48 \mathrm{~h}$ was analyzed by western blot ${ }^{*} P<0.05$ and ${ }^{*} P<0.01$ versus the NG EV group; ${ }^{*} P<$ 0.05 versus the HG EV group)

controlled by $\mathrm{p} 53$ and have a role in renal fibrosis ${ }^{16,21}$. Therefore, we analyzed the expression of these miRNAs in HG-stimulated and exendin-4-stimulated HK-2 cells, and determined that miR-192 was most significantly increased after HG treatment and repressed by exendin-4 (Fig. 4a). Furthermore, as shown in Fig. 4b, the expression of miR-192 was significantly downregulated in Ex-HG group-derived EVs compared with that in HG group-derived EVs. To confirm that exendin-4 regulated miR-192 expression through p53, we first used pifithrin-a to inhibit p53 expression. As shown in Fig. 4c, the expression of miR-192 was significantly downregulated in HG + pifithrin-a-treated HK-2 cells, indicating that HG regulated miR-192 expression in a p53-dependent manner. We then investigated the underlying mechanism by which exendin-4 regulates miR-192. As shown in Fig. 4d and e, HG upregulated p53 expression, but this increase was reversed by exendin-4. Using a ChIP assay, we determined that HG promoted p53 binding to the promoter of miR-192, which was blocked by exendin-4 (Fig. 4f, g).

\section{Exendin-4 downregulates miR-192 expression induced by HG in recipient cells}

To determine whether miR-192 can be transported through EVs, EVs from the NG, HG, exendin-4, and ExHG groups were collected and co-cultured with HK-2 cells. As shown in Fig. 5a, miR-192 levels in recipient cells co-cultured with EVs from HG-treated cells were significantly upregulated compared with the levels in cells co-cultured with EVs from NG-treated cells, whereas levels were reduced in cells co-cultured with EVs from Ex-HG-treated cells compared with the levels in those treated with HG group-derived EVs. However, we did not 

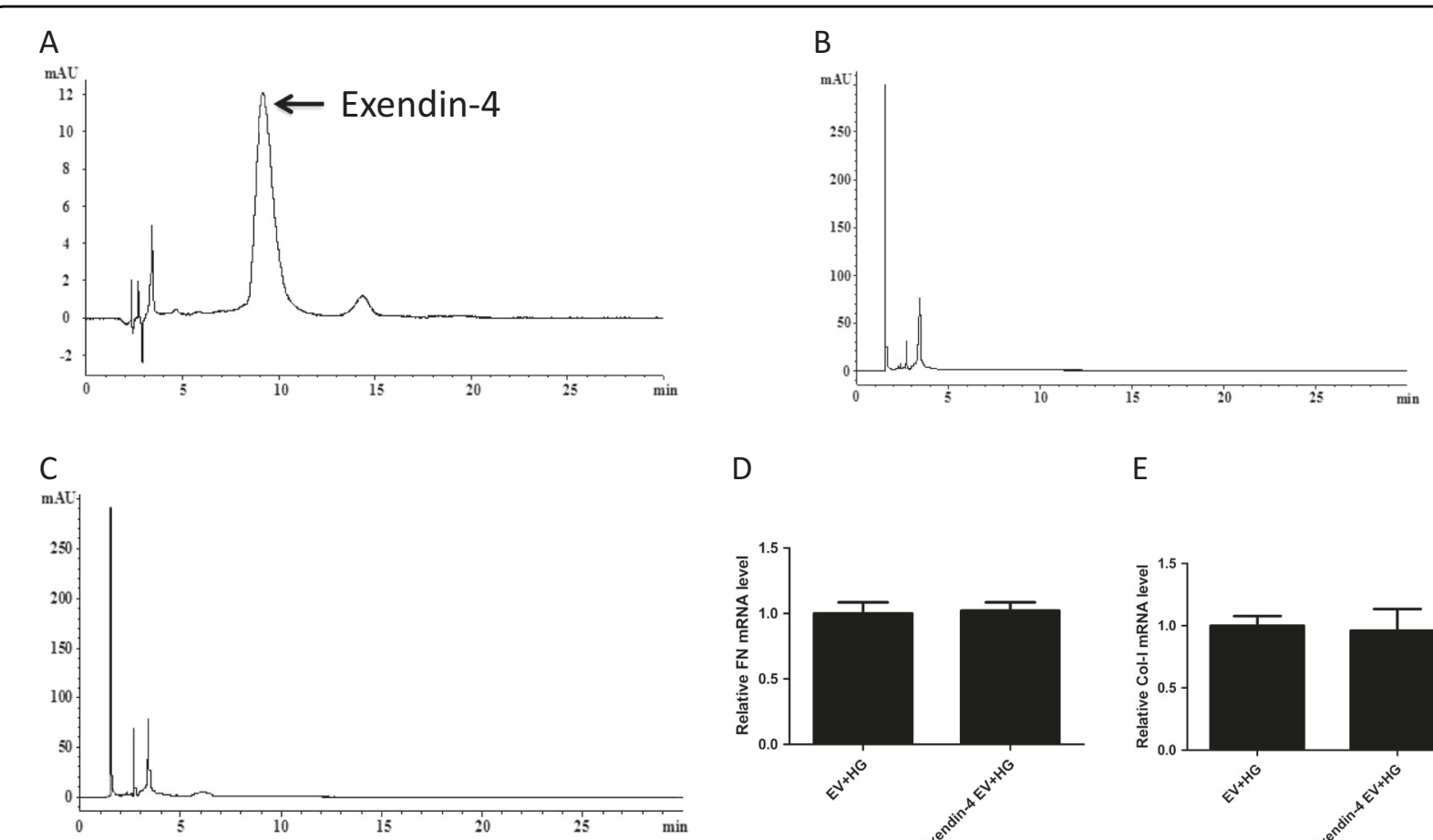

D

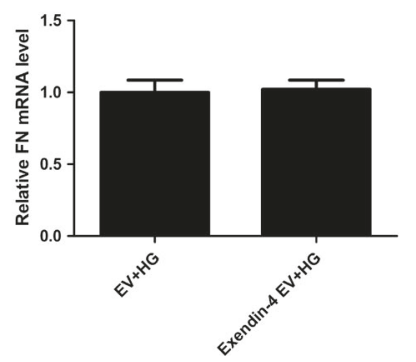

E

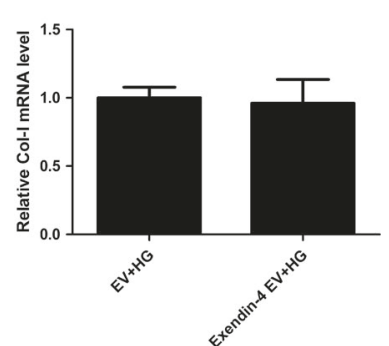

Fig. 3 Exendin-4 group-derived EVs loaded little exendin-4. a The HPLC chromatogram from the standard sample of exendin-4 in PBS (1 mg/ml). b The HPLC chromatogram from NG group-derived EVs. c The HPLC chromatogram from exendin-4 group-derived EVs. d, e Relative expression of Col-I and FN in HG-treated HK-2 cells co-cultured with exendin-4 group-derived EVs

observe a change in miR-192 levels in recipient cells cocultured with EVs from the exendin-4 group compared with the levels in those co-cultured with EVs from the NG group.

\section{EV-mediated miR-192 delivery into recipient cells promotes fibrosis}

To further explore the role of EV miR-192 in the promotion of renal fibrosis, we transfected HK-2 cells with a miR-192 inhibitor before HG treatment, collected the EVs from these cells, and co-cultured the EVs with recipient cells. As shown in Fig. 5b, the miR-192 inhibitor significantly downregulated miR-192 expression in EVs, and these EVs did not upregulate miR-192 expression in recipient cells (Fig. 5c). Furthermore, we analyzed FN and Col-1 expression in recipient cells and found that the miR-192 inhibitor group-derived EVs blocked FN and Col-I upregulation (Fig. $5 \mathrm{~d}-\mathrm{g}$ ). Thus, the fibrosis caused by the EVs derived from HG-treated cells may be induced by miR-192.

\section{miR-192 targets GLP1R in HK-2 cells}

To further explore the mechanism by which miR-192 regulates renal fibrosis, we used TargetScan to identify miR-192 target genes. Interestingly, GLP1R, a receptor of GLP-1, was identified as a target of miR-192 (Fig. 6a). We explored whether GLP1R is a target of miR-192. First, we transfected HK-2 cells with a miR-192 mimic and miR192 inhibitor to overexpress and downregulate miR-192 expression, respectively (Fig. 6b, c). On the basis of a luciferase reporter assay, the miR-192 mimic significantly decreased the 3'-UTR activity of GLP1R (Fig. 6d), whereas the miR-192 inhibitor increased the 3'-UTR activity of GLP1R (Fig. 6e). In addition, the miR-192 mimic aggravated the HG-induced decrease in the expression of GLP1R, whereas the miR-192 inhibitor reversed HGinduced GLP1R expression (Fig. 6f-k). Furthermore, EVs from HG-treated HK-2 cells also reduced GLP1R expression, and this effect was reversed by the EVs derived from the Ex-HG-treated cells (Fig. 6l-n).

\section{GLP1R knockdown induces renal fibrosis}

To evaluate the role of GLP1R in renal fibrosis, we transfected HK-2 cells with si-GLP1R. As shown in Fig. 7a, si-GLP1R-2 efficiently reduced GLP1R expression in HK-2 cells. Moreover, we evaluated the role of GLP1R in renal fibrosis and observed that si-GLP1R significantly upregulated FN and Col-I at both the mRNA and protein levels, which indicated that GLP1R has a role in renal fibrosis (Fig. 7b-f).

\section{miR-192 regulates renal fibrosis through GLP1R}

To explore whether miR-192 promotes renal fibrosis through GLP1R, we co-transfected HK-2 cells with both 
A

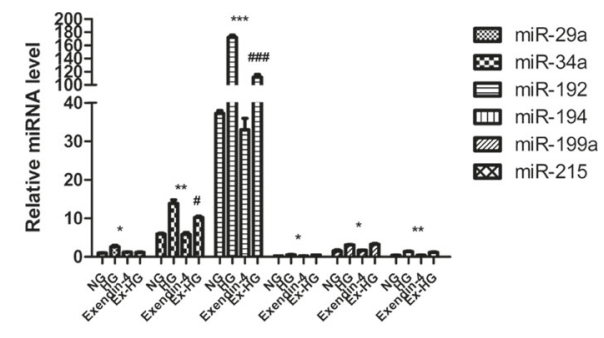

C

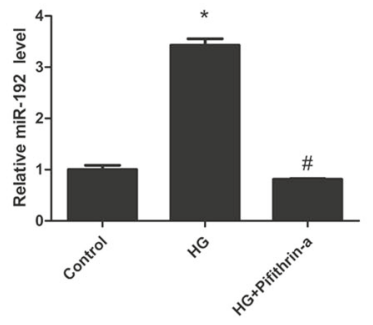

D

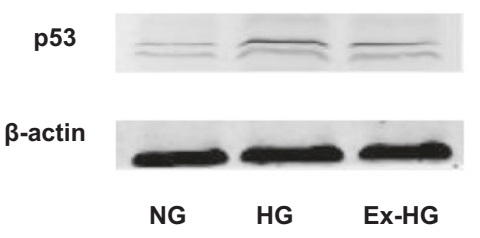

B

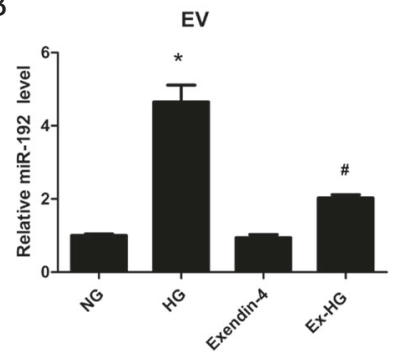

$\mathrm{E}$

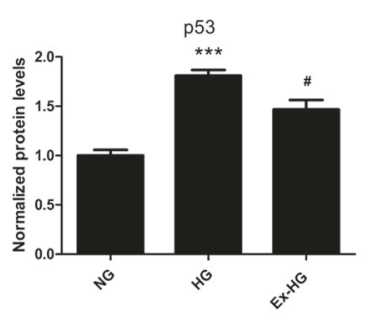

G

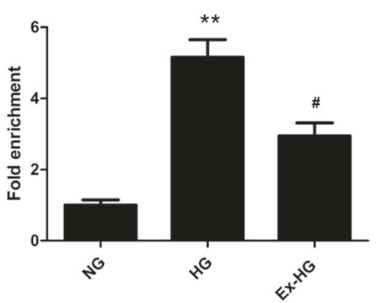

Fig. 4 Exendin-4 inhibited miR-192 expression via p53. a Effect of exendin-4 treatment on the expression of miRNA in HK-2 cells exposed to HG. b Effect of exendin-4 treatment on the expression miR-192 in EVs derived from HK-2 cells exposed to HG. $\mathbf{c}$ Effect of pifithrin-a treatment on the expression miR-192 in HK-2 cells exposed to HG. d, e Effect of exendin-4 treatment on the expression of p53. $\mathbf{f}$ Predicted p53-binding sites on the miR-192 promoter. $\mathbf{g}$ Exendin-4 inhibits the binding of p53 to the miR-192 promoter $\left({ }^{*} P<0.05\right.$, ${ }^{* *} P<0.01$, and ${ }^{* * *} P<0.001$ versus the NG group; ${ }^{\#} P<$ 0.05 versus the $\mathrm{HG}$ group)

si-GLP1R and a miR-192 inhibitor. As shown in Fig. 8a, the miR-192 inhibitor-induced GLP1R upregulation was blocked by si-GLP1R. As shown in Fig. 8b-g, amelioration of the renal fibrosis caused by the miR-192 inhibitor was abolished by si-GLP1R. These results indicate that miR192 aggravates renal fibrosis through a GLP1R-dependent manner.

\section{Discussion}

Exendin-4 has been shown to be beneficial for renal function by targeting GLP1R. In our study, we revealed a new mechanism by which exendin-4 can inhibit renal fibrosis. Specifically, exendin-4 downregulates cellular and secreted miR-192 expression induced by HG, thus inhibiting GLP1R downregulation in both donor and recipient cells (Fig. 9).

Recent studies have reported that exendin- 4 exerts a renal-protective role independent of blood glucose control. In a diabetic mouse model, administration of exendin-4 decreased urinary albumin and ameliorated glomerular hypertrophy without changing blood glucose levels $^{22}$. In our previous research, exendin-4 inhibited MC proliferation and FN secretion through AMPK activation ${ }^{13}$. In other research, exendin- 4 exerted a protective renal function by restoring nitric oxide production and renal blood flow ${ }^{23}$. In addition, exendin- 4 also improved renal function through activation of protein kinase $A^{24}$. However, the effect of exendin-4 on renal miRNA expression has not been previously studied. In recent years, studies have suggested that some medications can exert an anti-disease effect by regulating miRNA expression. Arunachalam et al. ${ }^{25}$ reported that metformin can downregulate microRNA-34a to prevent diabetic vascular disease. Atrasentan was found to prevent miR-199 expression, thereby preventing renal tubular injury ${ }^{26}$. Transcription factors are known to have a role in regulating miRNA expression ${ }^{27}$. Zhao et al. ${ }^{17}$ found that exendin-4 attenuated p53 expression after 3 days of treatment. As a transcription factor, p53 can regulate several miRNAs ${ }^{28}$. After analysis, we found that miR-192 


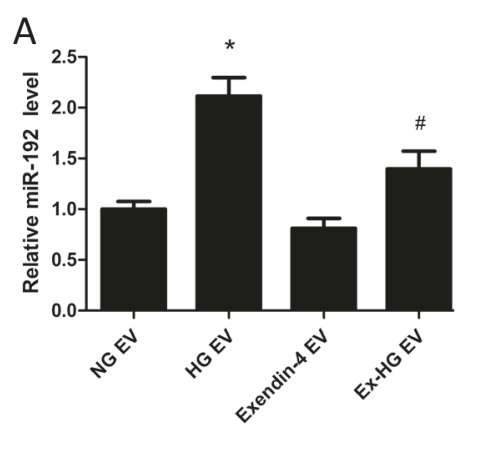

D

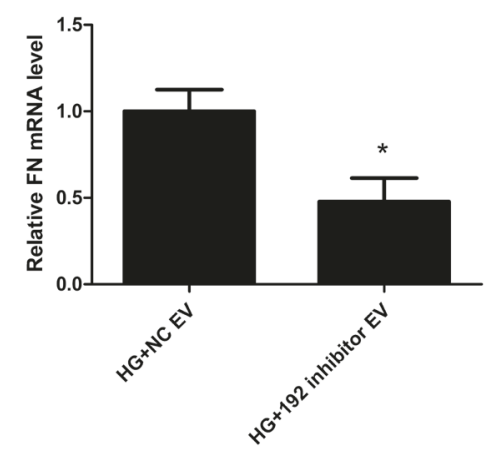

$\mathrm{F}$
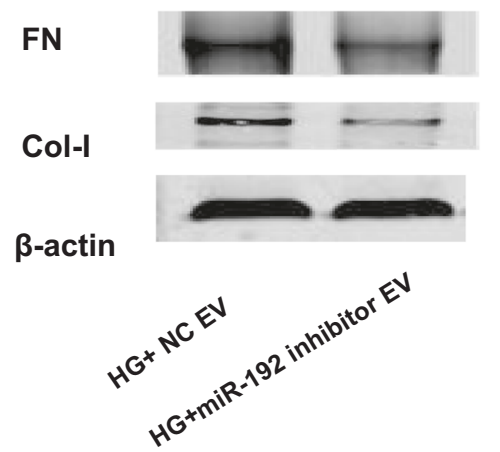

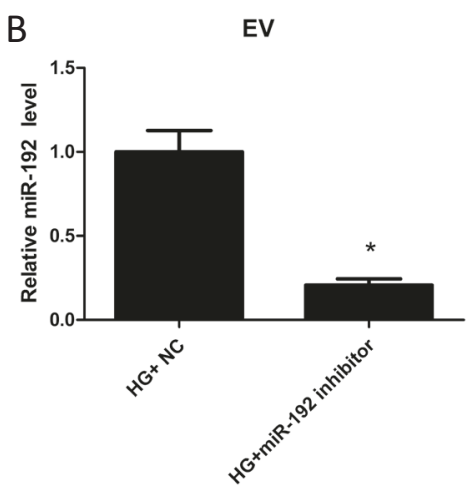

C

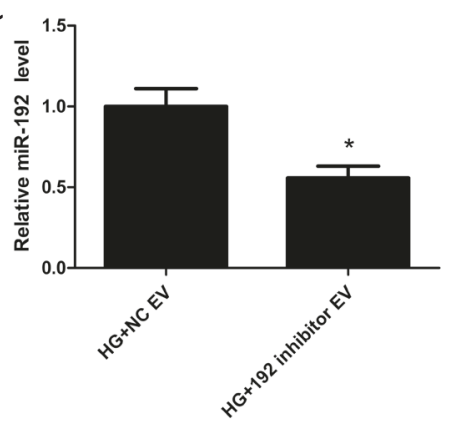

$\mathrm{E}$

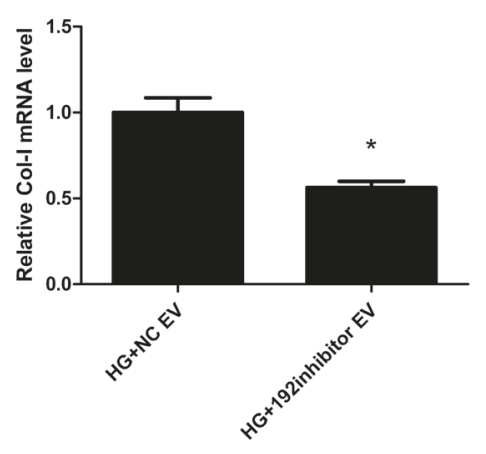

G

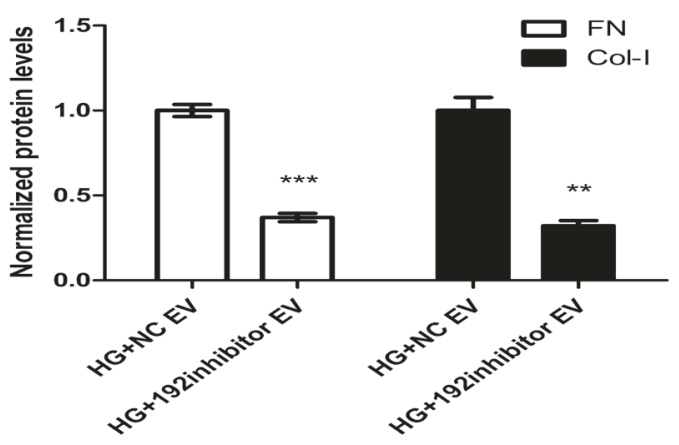

Fig. 5 miR-192 in donor-cell EVs induced fibrosis in recipient cells. a Relative expression of miR-192 in HK-2 cells after incubation with EVs derived from NG, HG, exendin-4, and Ex-HG groups for $48 \mathrm{~h}\left({ }^{*} P<0.05\right.$ versus the NG EV group; ${ }^{\#} P<0.05$ versus the HG EV group). $\mathbf{b}$ Relative expression of miR-192 in EVs derived from HK-2 cells pre-transfected with the miR-192 inhibitor or negative control (NC) and exposed to HG for $48 \mathrm{~h}$ ( ${ }^{*} P<0.05$ versus the HG + NC group). c-e Relative expression of miR-192, Col-I, and FN in HK-2 cells after incubation with EVs derived from HK-2 cells pre-transfected with the miR-192 inhibitor or NC and exposed to HG for $48 \mathrm{~h}$. f, $\mathbf{g}$ Col-I and FN expression in HK-2 cells after incubation with EVs derived from HK-2 cells pre-transfected with the miR-192 inhibitor or NC and exposed to HG for $48 \mathrm{~h}$ was analyzed by western blot ${ }^{*} P<0.05$ and ***P $<0.001$ versus the HG + NC EV group)

was most significantly increased after HG treatment and repressed by exendin-4 (Fig. 4a). To verify that HG regulates miR-192 expression in a p53-dependent manner, we treated cells with pifithrin-a, a p53 inhibitor, and HG and observed that the expression of miR-192 was significantly downregulated relative to the expression in HGtreated HK-2 cells. Furthermore, we observed that exendin-4 reversed HG-induced p53 expression (Fig. 4d, e). To explore the underlying mechanism, we used a ChIP assay to determine that HG promoted the binding of p53 to the promoter of miR-192 and that this effect was decreased by exendin-4 (Fig. 4f, g). These results indicate that exendin-4 regulates miR-192 expression during highglucose treatment in a p53-dependent manner. 


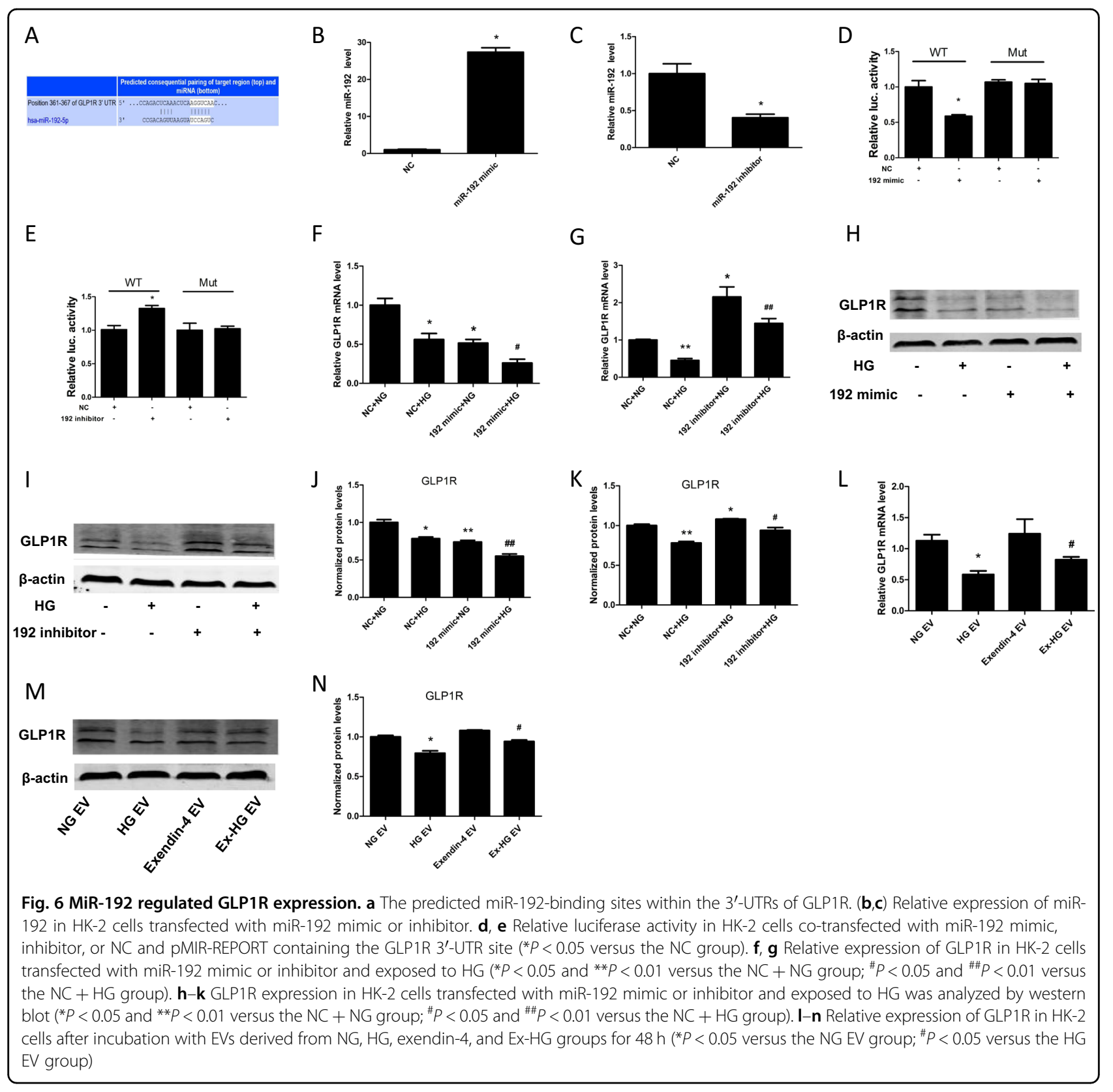

EVs have been reported to transfer information between cells and contribute to the development of disease. Borges et al. ${ }^{7}$ determined that exosomes released by injured epithelial cells carry TGF- $\beta 1$ mRNA, and promote proliferation and fibrosis in fibroblasts. Zhou et al. ${ }^{8}$ reported that TGF- $\beta$-treated tubular epithelial cells promote renal fibrosis by transferring miR-21 to recipient tubular epithelial cells via EVs. These studies proved that EVs can transport damage signals. Therefore, we sought to determine whether exendin- 4 can reduce the ability of injured renal tubular cells to transport damage signals to normal cells. First, we used Dil-C18-labeled EVs to confirm that the EVs entered HK-2 cells (Fig. 2d-f). Next, we measured
FN and Col-I expression after co-culture with EVs and determined that HG-treated HK-2 cell-derived EVs induced normal HK-2 cell fibrosis. However, Ex-HG group-derived EV treatment prevented fibrosis compared with HG group-derived EV treatment (Fig. $2 g-j$ ), leading us to conclude that exendin-4 weakens the transmission of this damage signal. Previous research has proven that many factors can affect EV release, and HG has been shown to increase the release of $\mathrm{EVs}^{29,}{ }^{30}$. Our research demonstrated that HG increased the total number of EVs but that Ex-HG decreased EV production compared with HG alone (Fig. 2c). Thus, exendin-4 may exert its effect by reducing the number of EVs. 
A

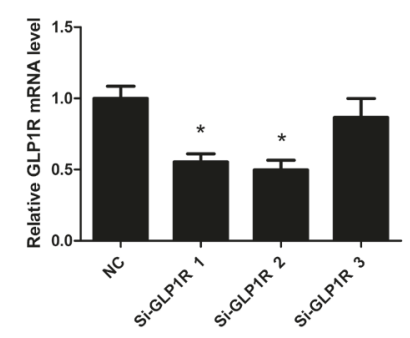

D

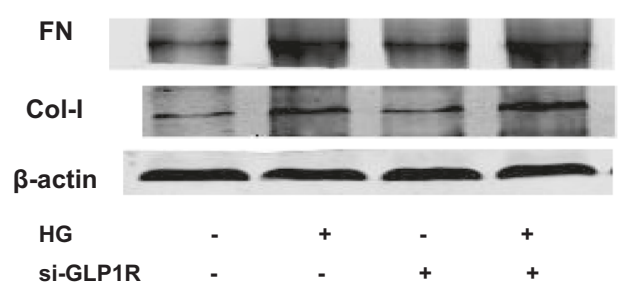

B

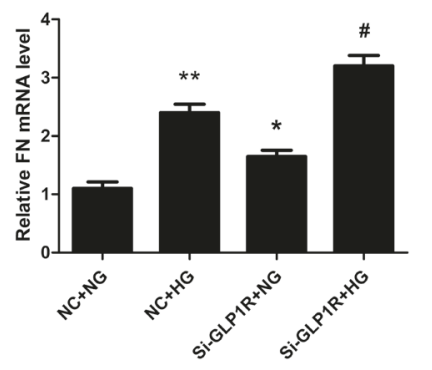

$\mathrm{E}$

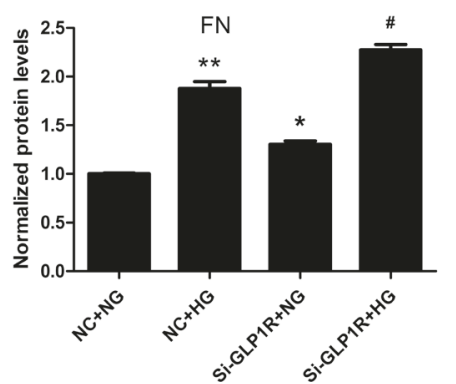

C
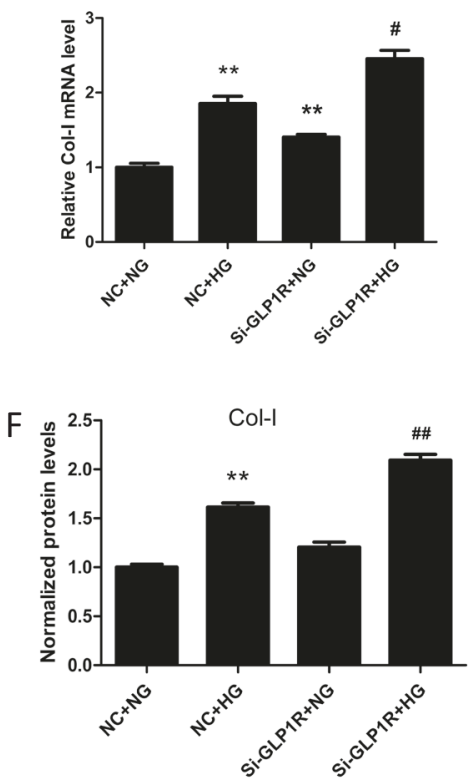

Fig. 7 GLP1R knockdown induced renal fibrosis. a The silencing ability of three si-GLP1R sequences in $H K-2$ cells $\left({ }^{*} P<0.05\right.$ versus the NC group). b, c Relative expression of Col-I and FN in HK-2 cells transfected with si-GLP1R and exposed to HG. d-f Col-I and FN expression in HK-2 cells transfected with si-GLP1R and exposed to HG was analyzed by western blot ${ }^{*} P<0.05$ and ${ }^{* *} P<0.01$ versus the NC $+\mathrm{NG}$ group; $P<0.05$ and ${ }^{\# \#} P<$ 0.01 versus the $\mathrm{NC}+\mathrm{HG}$ group)

EVs have been suggested to potentially be able to function as medication carriers. In one study, MSCs were shown to be able to package and deliver paclitaxel through $\mathrm{MVs}^{20}$. To explore whether the alleviated fibrosis effect of EVs from the Ex-HG group on HK-2 cells was mediated by exendin-4 contained in EVs, we measured the concentration of exendin-4 in EVs derived from exendin-4-treated HK-2 cells using HPLC. However, we determined that exendin-4 was not packaged in EVs, and EVs derived from exendin-4-treated HK-2 cells were unable to exert anti-fibrosis activity on HG-treated HK-2 cells. These observations indicate that little exendin- 4 was loaded into EVs derived from exendin-4-treated HK-2 cells. In recent years, several studies have reported that directly loading EVs, instead of loading EVs via the cell of origin, is much more effective. Active loading, in which electric pulses are used to increase the permeability of the $\mathrm{EVs}$, is more effective than merely incubating the isolated EVs with the therapeutic agent ${ }^{31}$. Thus, active loading may be a more effective method for the delivery of exendin-4 through EVs in future studies.

As miRNAs are reported to have a role in EV-mediated communication, we further explored the mechanism by which exendin-4 exerts its effect through EVs. We have reported that HG upregulates miR-192 expression in HK2 cell-derived $\mathrm{EVs}^{18}$. In the present study, we observed that miR-192 levels were downregulated in Ex-HG groupderived EVs compared with those in HG group-derived
EVs (Fig. 4b). To explore the role of miR-192, we used a miR-192 inhibitor to decrease miR-192 expression in EVs and observed that the EVs derived from miR-192 inhibitor-treated cells decreased FN and Col-1 expression compared with EVs from the HG group (Fig. $5 \mathrm{c}-\mathrm{g}$ ). Thus, our findings indicate that the EVs derived from HGtreated HK-2 cells induced renal fibrosis through the delivery of miR-192. Exendin-4 can decrease the levels of miR-192 in EVs, thereby blocking the transmission of signals from injured cells to normal cells. However, we observed that treatment of HK-2 cells with exendin- 4 was unable to downregulate miR-192 expression compared with control treatment (Fig. 4a). Furthermore, we did not observe differences in EV miR-192 expression between the control group and the exendin-4 group (Fig. 4b). As shown in Fig. 5a, we observed no significant difference in miR-192 expression between HK-2 cells co-cultured with EVs from the NG group and those co-cultured with EVs from the exendin- 4 group. These results indicate that exendin-4 significantly reduces miR-192 expression under HG conditions but has limited effectiveness under untreated conditions. Indeed, the role of exendin-4 under untreated conditions differs from cell to cell. In Nagayama's study ${ }^{32}$, exendin-4 decreased ERK1/2 and JNK activation only under AngII stimulation. Zhao et al. ${ }^{17}$ also reported that exendin-4 was unable to downregulate p53 expression under untreated conditions. However, Wei et $\mathrm{al}^{33}$ reported that exendin-4 exerts direct protective 


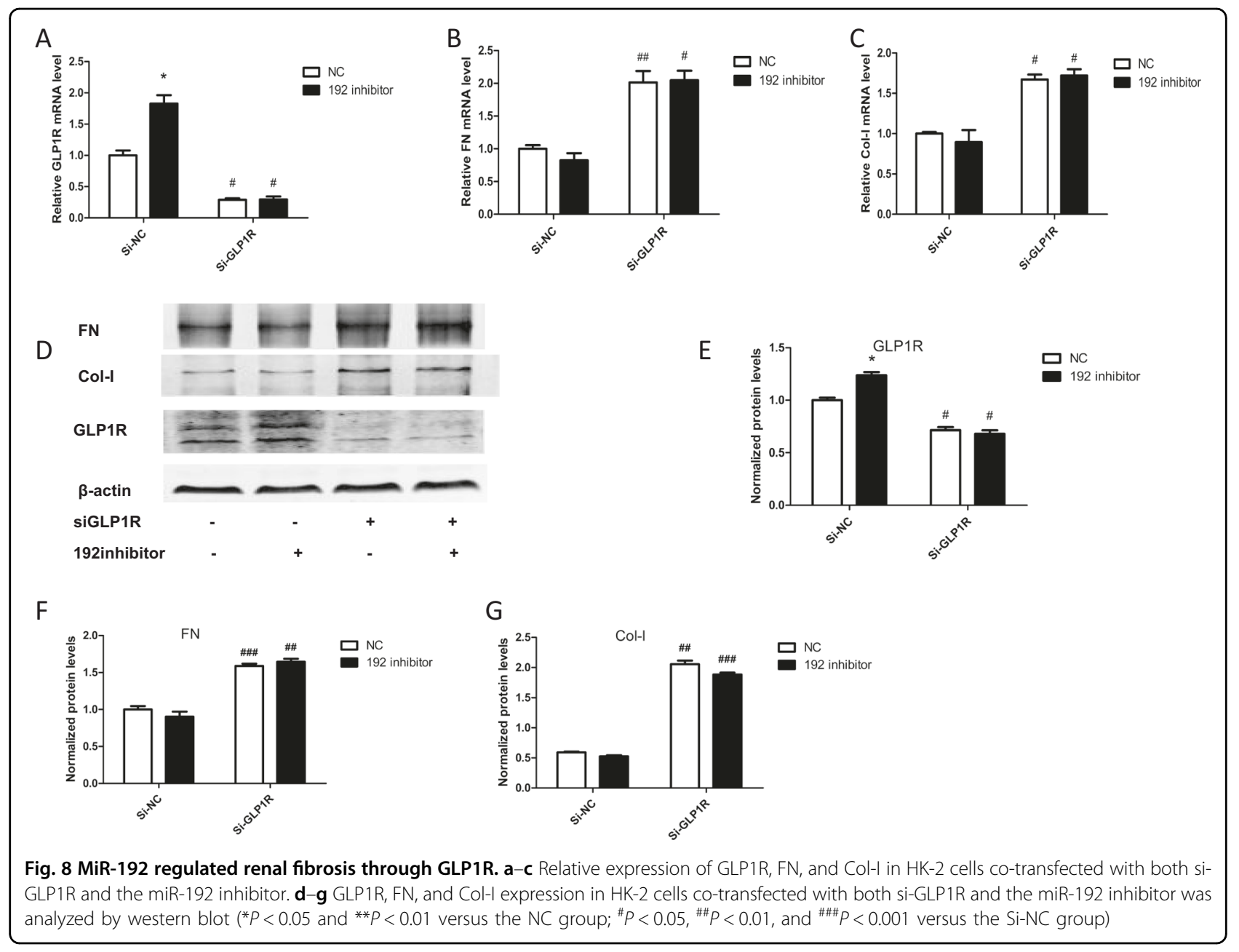

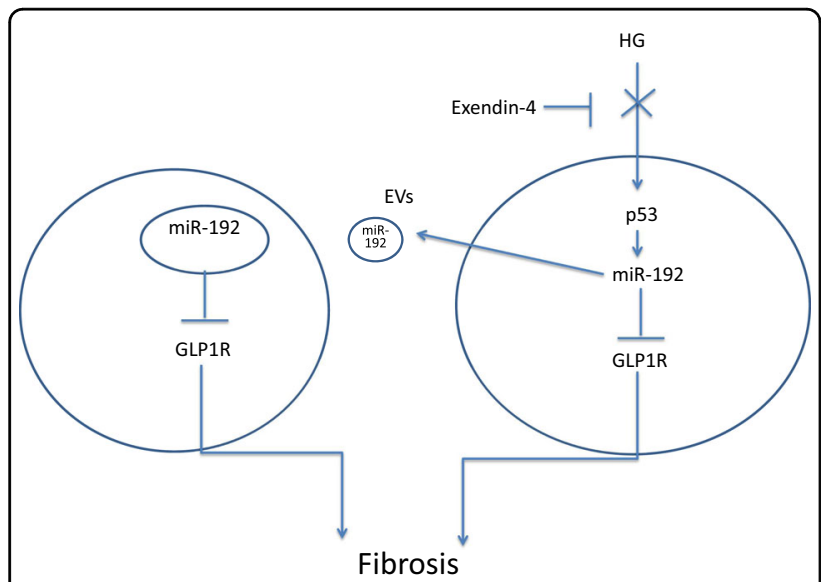

Fig. 9 Exendin- 4 inhibited the transfer of miR-192 and protected renal cells. Exendin-4 inhibited the transfer of EV miR-192 from HGinduced renal tubular epithelial cells to normal cells in a p53dependent manner, thus inhibiting GLP1R downregulation by miR192 and protecting renal cells effects on endothelial cells through the AMPK/Akt/eNOS pathway. Thus, further study is needed to explore the mechanism by which exendin-4 exerts its protective effects.

Although miR-192 expression during DKD development remains controversial ${ }^{34}$, using TargetScan, we found that miR-192 may target GLP1R to aggravate renal fibrosis (Fig. 6a). We showed that miR-192 targets GLP1R by binding to its 3 '-UTR and that overexpression of miR192 reduced GLP1R levels (Fig. 6b-k). To verify that miR192 promotes renal fibrosis through GLP1R, we cotransfected HK-2 cells with both si-GLP1R and a miR-192 inhibitor. The results showed that the amelioration of renal fibrosis caused by the miR-192 inhibitor was abolished by si-GLP1R (Fig. 8a-g). Thus, GLP1R is a key factor in the regulation of fibrosis by miR-192. However, in this study, we found that miR-192 altered both GLP1R protein and mRNA levels. Furthermore, the effect of miR192 on mRNA transcription was greater than that on its 
protein levels. Therefore, miR-192 may exert its effects not only by directly targeting GLP1R but also through some other indirect mechanisms. One possible mechanism is that miR-192 could directly target SLC39A6 (Zip6) $^{35}$, which has been identified as a putative GLP1Rinteracting protein ${ }^{36}$. Alternatively, miR-192 may positively interact with TGF- $\beta^{37}$, which could downregulate the AMPK pathway and thereby decrease GLP1R expression $^{38}$.

GLP1R is highly expressed in the kidney, especially in renal tubular epithelial cells ${ }^{39}$. The development of DKD is accompanied by decreased GLP1R levels, and exposure of renal cells to HG or lipotoxicity reduces GLP1R expression ${ }^{40}$. Furthermore, reduced GLP1R expression leads to renal cell autophagy ${ }^{39}$ and apoptosis ${ }^{38}$. Fibrosis is crucial in the development of DKD progression; however, the role of GLP1R in HK-2 cell fibrosis is unclear. As previously reported, HG significantly reduced GLP1R expression in HK-2 cells ${ }^{39}$. We also showed that knockdown of GLP1R induced FN and Col-1 expression (Fig. 7b-f). These results indicate that GLP1R loss not only leads to renal cell autophagy and apoptosis but also promotes fibrosis, thus accelerating DKD progression. GLP1R agonists have been reported to protect renal function via inhibition of $\mathrm{NAD}(\mathrm{P}) \mathrm{H}$ oxidase and cAMPPKA pathway activation ${ }^{41}$. However, in this study, we did not examine the mechanism by which GLP1R regulates renal fibrosis.

In conclusion, this research provides evidence that exendin-4 can inhibit the transfer of miR-192 from HGinduced renal tubular epithelial cells to normal cells, thus reversing GLP1R expression and protecting renal cells. These findings provide novel insight into the mechanism by which exendin-4 may be used to treat diabetic nephropathy.

\section{Acknowledgements}

This work was supported by research grants from the National Natural Science Foundation of China (Grant No. 81570724) and the Natural Science Foundation of Guangdong (Grant No. 2014A030313290).

\section{Conflict of interest}

The authors declare that they have no conflict of interest.

\section{Publisher's note}

Springer Nature remains neutral with regard to jurisdictional claims inpublished maps and institutional affiliations.

Received: 1 August 2017 Revised: 24 December 2017 Accepted: 7 February 2018.

Published online: 1 May 2018

\footnotetext{
References

1. Reidy, K., Kang, H. M., Hostetter, T. \& Susztak, K. Molecular mechanisms of diabetic kidney disease. J. Clin. Invest. 124, 2333-2340 (2014).
}

2. Bielesz, B. et al. Epithelial Notch signaling regulates interstitial fibrosis development in the kidneys of mice and humans. J. Clin. Invest. 120, 4040-4054 (2010).

3. Zheng, Z. et al. The coordinated roles of miR-26a and miR-30c in regulating TGFbeta1-induced epithelial-to-mesenchymal transition in diabetic nephropathy. Sci. Rep. 6, 37492 (2016)

4. Hao, J. et al. High-fat diet causes increased serum insulin and glucose which synergistically lead to renal tubular lipid deposition and extracellular matrix accumulation. Br. J. Nutr. 107, 74-85 (2012).

5. Stephan, J. P. et al. Albumin stimulates the accumulation of extracellular matrix in renal tubular epithelial cells. Am. J. Nephrol. 24, 14-19 (2004).

6. Wang, B. et al. Transforming growth factor-beta1-mediated renal fibrosis is dependent on the regulation of transforming growth factor receptor 1 expression by let-7b. Kidney Int. 85, 352-361 (2014).

7. Borges, F. T. et al. TGF-beta1-containing exosomes from injured epithelial cells activate fibroblasts to initiate tissue regenerative responses and fibrosis. J. Am. Soc. Nephrol. 24, 385-392 (2013).

8. Zhou, Y. et al. miR-21-containing microvesicles from injured tubular epithelial cells promote tubular phenotype transition by targeting PTEN protein. Am. J. Pathol. 183, 1183-1196 (2013).

9. Loyer, X., Vion, A. C., Tedgui, A. \& Boulanger, C. M. Microvesicles as cell-cell messengers in cardiovascular diseases. Circ. Res. 114, 345-353 (2014).

10. Raab-Traub, N. \& Dittmer, D. P. Viral effects on the content and function of extracellular vesicles. Nat. Rev. Microbiol. 15, 559-572 (2017).

11. van den Boorn, J. G., Dassler, J., Coch, C., Schlee, M. \& Hartmann, G. Exosomes as nucleic acid nanocarriers. Adv. Drug Deliv. Rev. 65, 331-335 (2013).

12. Fang, D. Y., King, H. W., Li, J. Y. \& Gleadle, J. M. Exosomes and the kidney: blaming the messenger. Nephrology 18, 1-10 (2013).

13. Xu, W. W. et al. Exendin-4 alleviates high glucose-induced rat mesangial cell dysfunction through the AMPK pathway. Cell. Physiol. Biochem. 33, 423-432 (2014).

14. Korner, M., Stockli, M., Waser, B. \& Reubi, J. C. GLP-1 receptor expression in human tumors and human normal tissues: potential for in vivo targeting. J. Nucl. Med. 48, 736-743 (2007).

15. Lee, I. S. et al. Exenatide reverses dysregulated microRNAs in high-fat dietinduced obese mice. Obes. Res. Clin. Pract. 10, 315-326 (2016).

16. Deshpande, S. D. et al. Transforming growth factor-beta-induced cross talk between p53 and a microRNA in the pathogenesis of diabetic nephropathy. Diabetes 62, 3151-3162 (2013).

17. Zhao, L., Li, A. Q., Zhou, T. F., Zhang, M. Q. \& Qin, X. M. Exendin-4 alleviates angiotensin II-induced senescence in vascular smooth muscle cells by inhibiting Rac1 activation via a cAMP/PKA-dependent pathway. Am. J. Physiol. Cell Physiol. 307, C1130-C1141 (2014).

18. Jia, Y. et al. miRNAs in urine extracellular vesicles as predictors of early-stage diabetic nephropathy. J. Diabetes Res. 2016, 7932765 (2016).

19. Pichiorri, F. et al. Downregulation of p53-inducible microRNAs 192, 194, and 215 impairs the p53/MDM2 autoregulatory loop in multiple myeloma development. Cancer Cell. 18, 367-381 (2010).

20. Pascucci, L. et al. Paclitaxel is incorporated by mesenchymal stromal cells and released in exosomes that inhibit in vitro tumor growth: a new approach for drug delivery. J. Control. Release 192, 262-270 (2014).

21. Chen, B. F., Suen, Y. K, Gu, S., Li, L. \& Chan, W. Y. A miR-199a/miR-214 selfregulatory network via PSMD10, TP53 and DNMT1 in testicular germ cell tumor. Sci. Rep. 4, 6413 (2014).

22. Sancar-Bas, S., Gezginci-Oktayoglu, S. \& Bolkent, S. Exendin-4 attenuates renal tubular injury by decreasing oxidative stress and inflammation in streptozotocin-induced diabetic mice. Growth Factors 33, 419-429 (2015).

23. Jensen, E. P. et al. Activation of GLP-1 receptors on vascular smooth muscle cells reduces the autoregulatory response in afferent arterioles and increases renal blood flow. Am. J. Physiol. Renal Physiol. 308, F867-F877 (2015).

24. Li, W. et al. Inhibition of the expression of TGF-betal and CTGF in human mesangial cells by exendin-4, a glucagon-like peptide-1 receptor agonist. Cell. Physiol. Biochem. 30, 749-757 (2012).

25. Arunachalam, G., Lakshmanan, A. P., Samuel, S. M., Triggle, C. R. \& Ding, H. Molecular interplay between microRNA-34a and Sirtuin1 in hyperglycemiamediated impaired angiogenesis in endothelial cells: effects of metformin. $J$. Pharmacol. Exp. Ther. 356, 314-323 (2016).

26. Kang, W. L. \& Xu, G. S. Atrasentan increased the expression of klotho by mediating miR-199b-5p and prevented renal tubular injury in diabetic nephropathy. Sci. Rep. 6, 19979 (2016). 
27. Zhang, H. M. et al. Transcription factor and microRNA co-regulatory loops: important regulatory motifs in biological processes and diseases. Brief Bioinform. 16, 45-58 (2015)

28. Goeman, F., Strano, S. \& Blandino, G. MicroRNAs as key effectors in the p53 network. Int. Rev. Cell Mol. Biol. 333, 51-90 (2017).

29. Hedlund, M., Nagaeva, O., Kargl, D., Baranov, V. \& Mincheva-Nilsson, L. Thermaland oxidative stress causes enhanced release of NKG2D ligand-bearing immunosuppressive exosomes in leukemia/lymphoma $T$ and B cells. PLoS ONE 6, e16899 (2011).

30. Wu, X. M., Gao, Y. B., Cui, F. Q. \& Zhang, N. Exosomes from high glucosetreated glomerular endothelial cells activate mesangial cells to promote renal fibrosis. Biol. Open 5, 484-491 (2016).

31. Ingato, D., Lee, J. U., Sim, S. J. \& Kwon, Y. J. Good things come in small packages: overcoming challenges to harness extracellular vesicles for therapeutic delivery. J. Control. Release 241, 174-185 (2016).

32. Nagayama, K. et al. Exendin-4 prevents vascular smooth muscle cell proliferation and migration by angiotensin II via the inhibition of ERK1/2 and JNK signaling pathways. PLOS ONE 10, e0137960 (2015).

33. Wei, R. et al. Exenatide exerts direct protective effects on endothelial cells through the AMPK/Akt/eNOS pathway in a GLP-1 receptor-dependent manner. Am. J. Physiol. Endocrinol. Metab. 310, E947-E957 (2016).
34. Kasinath, B. S. \& Feliers, D. The complex world of kidney microRNAs. Kidney Int 80, 334-337 (2011).

35. Lian, J. et al. miR-192, a prognostic indicator, targets the SLC39A6/SNAIL pathway to reduce tumor metastasis in human hepatocellular carcinoma. Oncotarget 7, 2672-2683 (2016).

36. Liu, Y. et al. Characterization of zinc influx transporters (ZIPs) in pancreatic beta cells: roles in regulating cytosolic zinc homeostasis and insulin secretion. J. Biol. Chem. 290, 18757-18769 (2015).

37. Kim, J. H., Lee, C. H. \& Lee, S. W. Hepatitis C virus infection stimulates transforming growth factor-beta1 expression through up-regulating miR-192. J. Microbiol. 54, 520-526 (2016).

38. Kim, D. I., Park, M. J., Heo, Y. R. \& Park, S. H. Metformin ameliorates lipotoxicityinduced mesangial cell apoptosis partly via upregulation of glucagon like peptide-1 receptor (GLP1R). Arch. Biochem. Biophys. 584, 90-97 (2015).

39. Zhao, X. et al. Liraglutide inhibits autophagy and apoptosis induced by high glucose through GLP1R in renal tubular epithelial cells. Int. J. Mol. Med. 35, 684-692 (2015).

40. Yin, Q. H. et al. Exendin-4 ameliorates lipotoxicity-induced glomerular endothelial cell injury by improving ABC transporter A1-mediated cholesterol efflux in diabetic apoE knockout mice. J. Biol. Chem. 291, 26487-26501 (2016).

41. Fujita, H. et al. The protective roles of GLP1R signaling in diabetic nephropathy: possible mechanism and therapeutic potential. Kidney Int. 85, 579-589 (2014). 